fächerte „Einstieg“, der dem Leser vermittelt wird, zu den unbestreitbaren Vorteilen des Buches. Noch nützlicher wäre der Band, wenn man den einzelnen Abschnitten weiterführende Literatur- und Quellenhinweise beigefügt hätte. So ist - alles in allem -, wer das Buch aus der Hand legt, beileibe noch kein Experte. Qualitativ wie quantitativ dürfte die Realität seinem Lateinamerika-Bild noch manche Korrektur aufnötigen. Dies als Herausforderung $\mathrm{zu}$ intensiverem Engagement zu begreifen, ist denn auch der wertvollste Impuls des Lateinamerika - PLOETZ.

Karl Hernekamp

Alois Móller

\title{
Städtische Marginalität und Militärregierung in Peru
}

Ludwigsburg, Eichhorn-Verlag Kallenberg 1977, kart., 510 S., 39,50 DM.

Die vorliegende Arbeit ist eine Göttinger Dissertation, die im Rahmen des Lehr- und Forschungsschwerpunktes ,Militär und Gesellschaft in peripheren Gesellschaften“ der Abteilung internationale Beziehungen der Göttinger Universität entstanden ist. Ihr Autor, der schon für seine Forschungsarbeit längere Feldaufenthalte in Peru hinter sich brachte, arbeitet heute als ILO-Berater in Peru. Möller ist sich bewußt, daß eine Beurteilung der Leistungen der Militärregierung nur im Rahmen einer Expertise der ökonomischen und politischen Bedeutung nichtkapitalistischer Produktionsweisen in der Peripheriegesellschaft Peru adäquat behandelt werden kann. Denn es ist wichtig, die ökonomischen Aktivitäten, die mit geringem Einkommen verbunden sind, in ihrer strukturellen Totalität zu analysieren. Erst auf diesem Hintergrund kann festgestellt werden, ob die Politik der Militärregierung strukturelle zur Uberwindung von Unterentwicklung beitragende Veränderungen hat realisieren können. Das Forschungsinteresse des Verfassers ist nicht appellativen Charakters; er will keine Vorschläge ausarbeiten, die eine beratende Funktion hätten. Vielmehr will er genetisch auf eine Komponente der Struktur der Unterentwicklung, nämlich die Beschäftigungsstruktur, eingehen und deren Veränderungsprozesse analysieren.

Möller beschränkt sich nicht darauf, die dualismus- und dependenztheoretischen Konzepte sachkundig zu untersuchen. Er steht zwar dem dependenztheoretischen Ansatz nahe, will ihn jedoch auf dessen empirische Validität befragen und dessen Eklektizismus überwinden. Er geht von der Annahme aus, daß in einer Peripheriegesellschaft eine vorkapitalistische Produktionsweise dominiert, deren Charakteristikum die Einheit der tätigen Menschen mit den Bedingungen ihrer materiellen Existenz ist. Durch die koloniale Penetration werden die Strukturen dieser Produktionsweise aufgelöst; es entstehen dann nichtkapitalistische Produktionsweisen, die innerhalb einer strukturell heterogenen Gesellschaftsformation integriert sind. Hier wäre wissenschaftlich primär zu untersuchen, welcher Sektor dominant ist bzw. ob diese nichtkapitalistischen Produktionsweisen von einer kapitalistischen Produktionsweise dominiert werden. Diese Frage kann nach Möller nur empirisch beantwortet werden, um dann auf einer niedrigen Stufe der Abstraktion zunächst auf mittlerer Ebene generalisierbare Aussagen zu treffen.

Der Hauptteil der Arbeit besteht aus einer sozialgeschichtlichen und sozialstrukturellen Untersuchung der peruanischen Gesellschaft. Zunächst stellt Möller fest, daß die Inka-Gesellschaft Merkmale der Asiatischen Produktionsweise aufweist, die er als eine vorkapitalistische Produktionsweise charakterisiert. Die spanischen Eroberer bedienten sich bestehender sozialer Organisationsformen, so daß die vorkapitalistischen Produktionsweisen weiterhin nach der kolonialen Eroberung dominant blieben. Erst gegen Ende des 19. Jahrhunderts leitete die Erschließung der natürlichen Ressourcen des Landes (Bergbau, Erdöl, Zucker) den 
Auflösungsprozeß der bisherigen Sozialstrukturen ein. Doch hatte der sich nunmehr exportorientierte Sektor nicht ausgeweitet; er hatte eine zentrale Enklaveneigenschaft, so daß der nunmehr sich entfaltende kapitalistische Sektor nicht dominant werden konnte. Doch das größte Problem der Enklavenwirtschaft bestand darin, mit Arbeitskräften versorgt zu werden, da Sklaven- und andere Formen der Zwangsarbeit damals noch nicht existierten (cf. 56 f.). Erst seit dem Zweiten Weltkrieg kann von einer dominanten kapitalistischen Gesellschaftsformation in Peru gesprochen werden, da seit diesem Zeitpunkt die vorkapitalistischen Produktionsweisen sich in einem Auflösungsprozeß befanden (cf. 59, 63). Es wird nunmehr für den Markt primär produziert, wenngleich eine geringe Beschäftigung möglich war, d. h., daß nur ein kleiner Teil der Arbeitskräfte in die kapitalistische Produktionsweise integriert war (cf. 81). Möller stellt aufgrund seines empirischen Materials fest, daß nur wenige Aussagen über eine industriekapitalistische Gesellschaft auf Peru zutreffen, daß diese Aussagen auch noch modifiziert werden müßten (cf. 93).

Möller charakterisiert die von ihm analysierte Sozialstruktur als eine blockierte Úbergangsform, die dominant kapitalistisch ist. Er geht dann ausführlich auf deren Auswirkungen auf den peruanischen Akkumulationsprozeß ein. Auch untersucht er die Marktpreisbildung innerhalb verschiedener Branchen der peruanischen Industrie und versucht, deren Auswirkungen analytisch zu ermitteln (130 ff.).

Diese Vorarbeiten ermöglichen Möller, dann die Beschäftigungsstruktur in der peruanischen Gesellschaft zu untersuchen. Er stellt zunächst fest, daß der Marxsche Begriff der ,,industriellen Reservearmee“ weiter entfaltet werden muß, um die Empirie Lateinamerikas analytisch zu fassen. In diesem Kontext diskutiert er dann ausführlich den Begriff der ,, marginalen Masse", der in der Dependenztheorie entwickelt worden ist. Das Einkommensniveau der Beschäftigten hängt von der sozialstrukturellen Entwicklung ab.

„Die Dynamik der kapitalistischen Entwicklung zerstört die vorkapitalistischen, schafft aber gleichzeitig nichtkapitalistische Produktionsweisen; sie bestimmt das Einkommensniveau in diesen Bereichen“" (p. 147). Das ist eine der zentralen Aussagen der Möllerschen Dissertation.

Möller legt in seiner Arbeit auch eine fundierte Analyse der Klassenstruktur Perus vor, die begrifflich stringent und empirisch fundiert ist. Er schlägt vor, ,,den Begriff der ,Marginalität' durch den der, nichtkapitalistischen Formen der Produktion und Distribution' zu ersetzen“ (191).

Nachdem Möller die diskutierten begrifflichen und empirischen Vorarbeiten geleistet hat, ist er nun in der Lage, die Gesellschaftspolitik der seit 1968 herrschenden Militärregierung zu würdigen und sie in die Totalität der peruanischen Gesellschaft einzuordnen. Möller formuliert die These: ,Die Politik der Strukturreformen und die Forcierung der ökonomischen Entwicklung sollen einer sozialen Revolution vorbeugen“ (287). Die Veränderungen, die eine Realisierung dieser Politik möglich machten, waren ,,Veränderung der Eigentumsverhältnisse und der Ausweitung der Produktion in den Exportsektoren und den Grundstoffindustrien“ (299). Diese werden im einzelnen untersucht. Der Autor will zunächst die Veränderungen in den nichtkapitalistischen Produktionsweisen und deren sozialen Charakter analysieren, was ihm ermöglicht, die staatliche Politik zu beurteilen. Der Staat dient der Militärregierung als Instrument der Veränderung durch Interventionen. Der Rahmen der Analyse sind die Interaktionen, die zwischen Staat, nationaler Wirtschaft und Weltmarkt stattfinden. Eine Gesamtwürdigung der Gesellschaftspolitik der peruanischen Militärregierung führt zu der Aussage, daß die Realisierung des Konzeptes der sozialen Mobilisierung, das verfolgt wurde, als gescheitert erklärt werden muß. Dies gilt auch für die Pläne ökonomischer und sozialer Reformen (cf. 331). 
Die Auflösung der vorkapitalistischen Sozialstrukturen bringt eine Gesellschaftsformation hervor, deren Úbergang strukturell blockiert ist: Es entfalten sich nunmehr Produktionsweisen, die nichtkapitalistisch sind, jedoch von einer kapitalistischen dominiert werden. Diese strukturell blockierte Entwicklung bildet den Gegenstand der Arbeit von Möller, d. h., daß er die ungenügende Integration der Arbeitskräfte im kapitalistischen Sektor untersucht. Eine Klassenanalyse der in nichtkapitalistischen Produktionsweisen angesiedelten Population hilft, diese Fragestellung näher zu beleuchten.

Eine Weiterentfaltung des Theorems der ,,industriellen Reservearmee“, eine Revision und Modifikation einiger der Thematik der vorliegenden Arbeit betreffenden Annahmen der Dependenztheorie gehören zu den wichtigen Leistungen der vorliegenden Publikation. Das empirische Material, zu dem die selbständige Verarbeitung noch bisher unerschlossenen statistischen Materials gehört, zählt auch zu den Vorzügen dieser Arbeit. Diese empirische Analyse ermöglicht auch die Entfaltung eines Periodisierungsversuches der Industrialisierung Perus.

In diesem Rahmen gehört die Einschätzung der sozialstrukturellen Veränderungsversuche der Militärregierung. Möllers These lautet: „,Die Entwicklung der Militärregierung zeigt, daß das ,radikalste' Projekt, das die Mittelklasse - oder zumindest deren militärische Fraktion - durchführen kann, der Staatskapitalismus ist" (386).

Kritisch ist anzumerken, daß Möller zwar sein umfangreiches empirisches Material begrifflich verarbeitet, es jedoch unterlassen hat, aus seiner Expertise theoretische Entwürfe zu entfalten. Dieser Sachverhalt scheint ihm bewußt zu sein, zumal er mehrmals betont, daß er auf einer niedrigen Stufe der Abstraktion bleiben will. Einen höheren Grad der Abstraktion hält er erst dann für möglich, wenn vorher auf der von ihm betriebenen Abstraktionsstufe ein validisierter begrifflicher Rahmen entfaltet worden ist.

Bassam Tibi 